\title{
M-Dwarf Multiplicity Rate in the Solar Vicinity
}

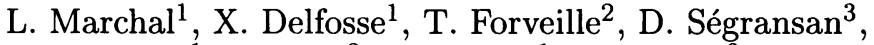 \\ J. L. Beuzit ${ }^{1}$, S. Udry ${ }^{3}$, C. Perrier ${ }^{1}$, M. Mayor ${ }^{3}$, J.-L. Halbwachs ${ }^{4}$ \\ (1) Laboratoire d'Astrophysique de Grenoble, France, (2) \\ Canada-France-Hawaii Telescope Corp., Hawaii, (3) Observatoire de \\ Genève, Switzerland, (4) Observatoire astronomique de Strasbourg, \\ France
}

\begin{abstract}
We present results based on a 6 year survey of a solar vicinity M-dwarf sample. We have obtained their multiplicity rate and orbital elements for the binaries. Such results could give us keys for a better understanding of stellar formation.
\end{abstract}

\section{Introduction and Sample}

This program started in September 1995 and searches for companions in a volume-limited sample of $\mathrm{M}$ dwarfs. Its goals are (1) multiplicity statistics for systems with an $\mathrm{M}$ dwarf primary: frequency of single and multiple stars, distribution of orbital elements (this article); (2) accurate masses for very low mass stars $\left(\mathrm{M}<0.6 \mathrm{M}_{\odot}\right.$, see Ségransan et al. 2000$)$, and an accurate mass-luminosity relation (Delfosse et al. 2000); and (3) identify substellar companions (Delfosse et al. 1998b) and rotational velocities of $\mathrm{M}$ dwarfs (see Delfosse et al. 1998). The sample has 104 systems, and it is volume limited for the spectral type M0-M5.5

\section{Observing Strategy and Analysis}

This programme combines two observational techniques, with overlapping sensitivity ranges that together cover all separations and (stellar) mass ratios: (1) radial-velocity monitoring: (sensitive to $P \leq$ a few dozen years) using ELODIE (1.93m-telescope at the Observatoire de Haute-Provence), and CORAVEL (Swiss telescope at Observatoire de Haute-Provence), and (2) adaptive optics imaging: (sensitive to $P \geq 1$ year) using PUEO (3.6m CFH telescope, Hawaii) and ADONIS (3.6m ESO telescope, Chile).

We have used Monte-Carlo simulations to obtain the detection rate expected for our observation set, and to correct, if needed, the observed distributions. Stellar companions: Our completeness is very close to $100 \%$, for all periods up to $10^{5}$ days (limited by the field of view of the adaptive optics images). For periods above $10^{5}$ days, we rely on common proper motion binaries from the literature, which is considered essentially complete for companions to nearby $\mathrm{M}$ dwarfs in that separation range (e.g. Hinz et al. 2002). Our sample is therefore complete for stellar companions, and so we can derive the multiplicity statistics without any correction. 
Brown dwarf secondaries: Our detection rate for $\mathrm{P}<10^{3}$ days (from radial velocities) is better than $75 \%$, depending on the period and mass ratio. We found no such brown dwarf, indicating that they are very rare. We have some brown dwarf candidates at larger separations (from adaptive optics), whose status needs to be clarified through establishment of common proper motion, or lack thereof.

\section{Conclusions}

- The multiplicity rate (for stellar companions) for $\mathrm{M}$ dwarfs is $31 \pm 5 \%$.

- The binary rate monotonously decreases with primary mass: $70 \%$ for OB stars (Preibish 2001), 57\% for G dwarfs (Duquennoy \& Mayor 1991), and 31\% for $\mathrm{M}$ dwarfs.

- The M and G dwarfs have very similar separation distributions for $d \leq 10 \mathrm{AU}$.

- $\mathrm{M}$ dwarfs have a companion deficit at larger separations relative to $\overline{\mathrm{G}} \mathrm{dwarfs}$. Dynamical disruption of wide M dwarf binaries (Sterzik \& Durisen 1998; Kroupa \& Buckert 2001)??

- For both G dwarfs (Tokovinin 2000; Halbwachs et al. 2002) and M dwarfs, short-period and long-period binaries have different mass ratio distributions: for $P>50$ days the distribution is consistent with a flat distribution, while shorter-period binaries have a strong preference for nearly equal masses. A Kolmogorov-Smirnov test shows that the two populations are clearly different, and suggests different formation/evolution mechanisms.

- Brown dwarfs companions of M dwarfs are very rare within $1.5 \mathrm{AU}$ (none found in 100 systems). This is analogous to the well-established brown dwarf desert around $\mathrm{G}$ dwarfs.

- As was found for $\mathrm{G}$ dwarfs by Tokovinin (2001), most M dwarf close binaries ( $P \leq 50$ days) belong to higher multiplicity systems.

- The mass ratio-eccentricity diagram shows that when mass ratio is near 1 , the orbit is circularized.

\section{References}

Delfosse, X., et al. 1998, A\&A, 331, 581

Delfosse, X., et al. 1998b, A\&A, 338, L67

Delfosse, X., et al. 2000, A\&A, 364, 217

Duquennoy, A., \& Mayor, M. 1991, A\&A, 248, 485

Hinz, J. L., et al., 2002, AJ,123, 2027

Kroupa, A., \& Burkert, A. 2001, ApJ,555, 945

Preibisch, T., Guenther, E., \& Zinnecker, H. 2001, AJ, 121,1040

Preibisch, T., Weigelt, G., \& Zinnecker, H. 2001, in IAU Symp. 200, 69

Ségransan et al., 2000, A\&A, 364, 665

Sterzik, M. F., \& Durisen, R. H. 1998, A\&A, 339, 95

Tokovinin, A. A. 2000, A\&A, 360, 997

Tokovinin, A. A. 2001, in IAU Symp. 200, 84 